\title{
The feasibility of organ procurement at a hospital-independent facility: A working model of efficiency
}

\author{
Nader Moazami, MD, ${ }^{\text {a }}$ Omid H. Javadi, MD, ${ }^{a}$ Dean F. Kappel, ${ }^{\mathrm{c}}$ Jessica Wagner, $\mathrm{RN}^{\mathrm{c}}{ }^{\mathrm{c}}$ and Martin D. Jendrisak, MD, ${ }^{\mathrm{b}}$ \\ St Louis, Mo
}

$\mathrm{M}$ ultiorgan procurement traditionally occurs in the hospital setting after declaration of brain death. The entire organ procurement process, which involves managing the donor, placing the organs (often at several different institutions), and retrieving the organs, is a complex task. ${ }^{1}$ Often this process is further complicated by the inability to proceed to the operating room in a timely fashion because of limitations related to staff availability and other scheduling difficulties at the donor hospital. Surveys indicate that nearly $20 \%$ of operating room nurses view organ procurement as burdensome when added to an already busy surgery schedule. ${ }^{2}$

Expeditious access to the operating room and avoidance of unnecessary delays related to the actual scheduling of organ procurement are important components of multiorgan procurement. In an effort to address these matters, Mid-America Transplant Services (MTS), the regional organ procurement organization that covers Missouri, Southern Illinois, and Northern Arkansas, constructed operating rooms in its corporate facilities. Donors from various regional and local hospitals are transported to this hospitalindependent facility for organ procurement. This nontraditional method of cadaver organ procurement, in a facility other than the usual hospital operating room setting, has been a unique approach. This report evaluates the safety and efficacy of this model for thoracic organ retrieval.

\section{Materials and Methods}

The facility consists of a 250 -sq $\mathrm{ft}$ preoperative unit adjacent to a 400-sq ft fully equipped operating room. The entire setup has undergone the necessary building modifications and is equipped to provide full operating room capabilities in compliance with all building codes and regulatory agency requirements for sterility and

From the Sections of Cardiothoracic Surgery ${ }^{\mathrm{a}}$ and Transplantation, ${ }^{\mathrm{b}}$ Department of Surgery, Washington University School of Medicine, and Mid-America Transplant Services, ${ }^{\mathrm{c}}$ St Louis, Mo.

D.F.K. is chief executive officer and president of Mid-America Transplant Services. J.W. is a transplant coordinator and employee of MidAmerica Transplant Services. M.D.J. is one of the medical directors of Mid-America Transplant Services. N.M. and O.H.J. have no affiliation with Mid-America Transplant Services.

Received for publication Aug 31, 2006; accepted for publication Dec 12, 2006.

Address for reprints: Nader Moazami, MD, 660 S Euclid Ave, Campus Box 8234, St Louis, MO, 63110-1013 (E-mail: moazamin@wustl.edu).

J Thorac Cardiovasc Surg 2007;133:1389-90

$0022-5223 / \$ 32.00$

Copyright () 2007 by The American Association for Thoracic Surgery doi:10.1016/j.jtcvs.2006.12.038 infection control. The site undergoes routine evaluation of organ procurements and outcomes to ensure compliance and safety.

Donor management is provided by MTS nurse coordinators under supervision of the medical directors. Intraoperative care is provided by an anesthesiologist. Criteria for transport to the MTS facilities include donor age older than 10 years, absence of active hemorrhage, hemodynamic stability with or without vasopressor support, and family consent.

From November 2001 to September 2004, a total of 123 donors were transferred from local and regional hospitals to the MTS facility. Standard donor management protocols were performed for all organ recoveries. All thoracic organ procurement data were compared with those from 80 retrievals performed during the same period at the local and regional hospitals within the same coverage area without transport to the MTS facilities. Statistical analysis was performed with the Student $t$ test.

\section{Results}

Mean ages were $30 \pm 15$ years for donors taken to MTS and $44 \pm$ 16 years for those at hospitals. There were no complications associated with transfer of the donors to MTS or during the procurement process. Overall time from brain death to crossclamping was decreased when donors were transferred to MTS (Table 1). Cold ischemic times were reduced for organs; this was more pronounced in comparison with regional hospitals (Table 2). Mean costs of organ procurement per donor were $\$ 17,000$ for hospitalbased recovery and $\$ 9000$ for $\operatorname{MTS}(P<.005)$.

\section{Discussion}

This report aims to highlight the feasibility and safety of conducting cadaveric organ recovery in an appropriately constructed and well-staffed hospital-independent facility. The impetus for development of this model was primarily the need to address the problem of timely access to the operating room and the ability to

TABLE 1. Time differences between groups at each stage of organ procurement

\begin{tabular}{lccc}
\hline & $\begin{array}{c}\text { Hospital } \\
(\mathbf{n}=\mathbf{1 2 3})\end{array}$ & $\begin{array}{c}\text { MTS } \\
(\mathbf{n}=\mathbf{8 0})\end{array}$ & $\begin{array}{c}\boldsymbol{P} \\
\text { value }\end{array}$ \\
\hline $\begin{array}{l}\text { Brain death to operating room } \\
\text { (min) }\end{array}$ & $810 \pm 345$ & $724 \pm 301$ & .07 \\
$\begin{array}{l}\text { Operating room to incision (min) } \\
\text { Incision to crossclamping (min) }\end{array}$ & $54 \pm 44$ & $93 \pm 85$ & $<.001$ \\
$\begin{array}{l}\text { Brain death to crossclamping } \\
\text { (min) }\end{array}$ & $966 \pm 379$ & $731 \pm 248$ & $<.001$ \\
& & &
\end{tabular}

All values are mean \pm SD. MTS, Mid-America Transplant Services; NS, not significant. 
TABLE 2. Cold ischemic times for hearts and lungs for all hospitals included in the analysis and for regional hospitals compared with those performed at MTS

\begin{tabular}{llcc}
\hline Cold ischemic time $(\min )$ & In hospital & At MTS & $\boldsymbol{P}$ value \\
\hline $\begin{array}{llll}\text { All hospitals } \\
\quad \text { Heart }(\mathrm{n}=72)\end{array}$ & $185 \pm 82$ & $146 \pm 57$ & $<.03$ \\
$\quad$ Lungs $(\mathrm{n}=61)$ & $178 \pm 106$ & $183 \pm 84$ & NS \\
Regional hospitals & & & \\
$\quad$ Heart ( $\mathrm{n}=40)$ & $221 \pm 86$ & $147 \pm 59$ & $<.01$ \\
Lungs $(\mathrm{n}=47)$ & $327 \pm 111$ & $192 \pm 64$ & $<.01$
\end{tabular}

All values are mean \pm SD. MTS, Mid-America Transplant Services; NS, not significant.

facilitate coordination of multiorgan procurement without external pressures from local hospitals.

Retrospective data from organ donors at the MTS facility relative to traditional in-hospital procurement suggest that donors can be safely transferred without any detrimental consequence to hemodynamic stability. The process leads to an overall reduction of time from brain death to crossclamping, increases in which may be detrimental to maintenance of donor stability and organ quality. ${ }^{3}$
Most of the time saved seems to lie in efficiency in getting the operation started once in the operating room. Furthermore, because most of the organs were delivered locally, cold ischemic times were significantly reduced. This was even more striking for more remote regional hospitals because of reduced traveling time and proximity of MTS to the transplanting centers.

In conclusion, this is a unique working model for organ recovery that would enhance ability to retrieve organs by bypassing the logistical difficulties of scheduling multiorgan retrieval at busy donor hospitals. Improved ability to coordinate timing, reduced ischemic times, and more a cost-efficient process make this an important model to be considered by other organ procurement organizations.

\section{References}

1. Bollinger RR, Heinrichs DR, Seem DL, Rosendale JD, Johnson KS UNOS Council for Organ Availability. United Network for Organ Sharing. Organ procurement organization (OPO), best practices. Clin Transplant. 2001;15 Suppl 6:16-21.

2. Van da Walker SG. The attitudes of operating room nurses about organ donation. J Transpl Coord. 1994;4:75-8.

3. Cantin B, Kwok BW, Chan MC, Valantine HA, Oyer PE, Robbins RC, et al. The impact of brain death on survival after heart transplantation: time is of the essence. Transplantation. 2003;76:1275-9. 